\title{
Effect of Deformation and Thermal Treatment of NiTi Alloy on Transition Sequence
}

\author{
H. Morawiec, D. Stróż and D. Chrobak \\ Institute of Physics and Chemistry of Metals, University of Silesia, 40-007 Katowice, Bankowa 12, \\ Poland
}

\begin{abstract}
Transformation sequences in a NiTi shape memory alloy after deformation and subsequent annealing were studied using various methods. Low temperature annealing of the deformed alloy caused that the R-phase preceeded the martensitic transformation. The R-phase formation during both cooling and heating depends strongly on the deformation degree and temperature of annealing. On DTA curves an additional peak in the martensitic transformation range was observed for specimens annealed at 500 and $550^{\circ} \mathrm{C}$. It is suggested that this peak was caused by presence of dislocation cell structure and subgrains whose boundaries interact with growing martensite plate.
\end{abstract}

\section{INTRODUCTION}

Deformation and subsequent annealing of NiTi alloys offers great possibilities of controlling temperatures and sequences of transformations. Todoroki and Tamura [1] showed that, depending on annealing temperature, changes in transformation sequences in deformed alloys occur during both heating and cooling. Abujudom et. al. [2] found that the deformation degree significantly influences both course and characteristic temperatures of the transformations. The authors of [3-8] studied the effect of both deformation degree and annealing temperatures on the course of transformations. In [4,5] and [8] the influence of deformation and annealing on shape recovery phenomenon was investigated. It was shown [8] that large deformations have a positive influence on shape recovery. However, the results mentioned above show certain discrepancies, especially in variations of characteristic temperatures versus annealing temperature and in sequences of transformations. The aim of this paper was to establish the influence of dislocation density and configuration on the characteristic temperatures and sequences of transformations.

\section{EXPERTMENTAL PROCEDURE}

A commercial NiTi shape memory alloy containing 50.6 at $\% \mathrm{Ni}$ was used in this study. Specimens in shape of strips were quenched from $800^{\circ} \mathrm{C}$ after annealing for $1 \mathrm{~h}$ and then cold-rolled by $5 \%, 10 \%, 20 \%$ or $25 \%$ reduction. The deformed samples were then annealed at various temperatures between $300-550^{\circ} \mathrm{C}$. The transformation behaviour and the characteristic temperatures were determined using the DTA method on the TA1 Mettler instrument and the internal friction method on an acoustic relaxator with the logarithmic decrement of damping.

The phase composition of the specimens was established using the X-ray phase analysis on a Philips diffractometer equipped with a temperature attachment. $\mathrm{CuK} \alpha$ radiation was used. The alloy structure was studied on thin foils using JEM 200B transmission electron microscope. 


\section{EXPERIMENTAL RESULTS}

\subsection{Effect of deformation}

The studied alloy, after quenching from $800^{\circ} \mathrm{C}$, consisted of the parent $\mathrm{B} 2$ phase only as was shown by $\mathrm{X}$-ray studies. Deformation by cold-rolling caused strengthening of the alloy as evidenced by increase in its hardness. The $110 \mathrm{~B} 2$ diffraction line widened due to increase in lattice strain and density of dislocations (Fig.1).

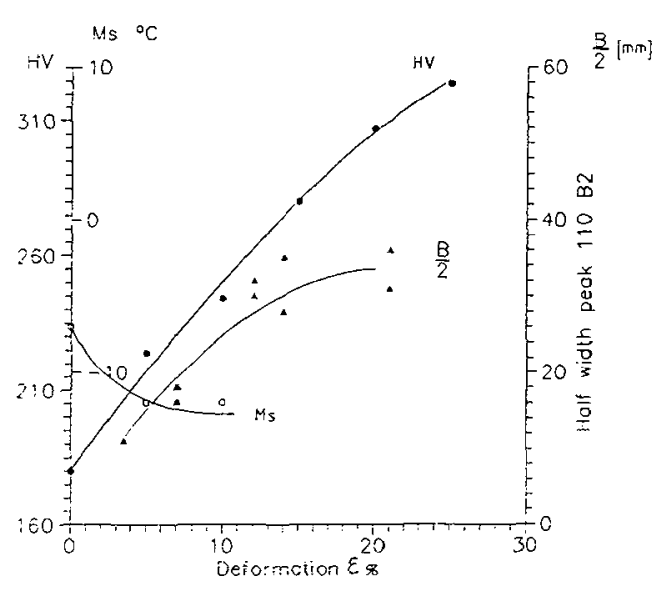

Fig. 1 Hardness, $M_{s}$ temperature and half-width of the $110 \mathrm{~B} 2$ versus deformation degree

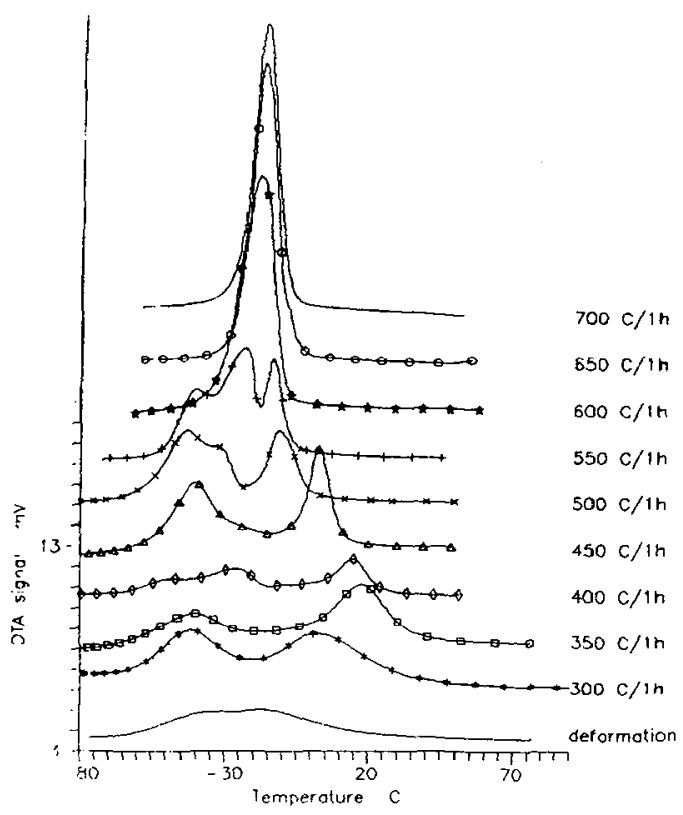

Fig. 3 The DTA curves obtained during cooling of the alloy deformed by $10 \%$

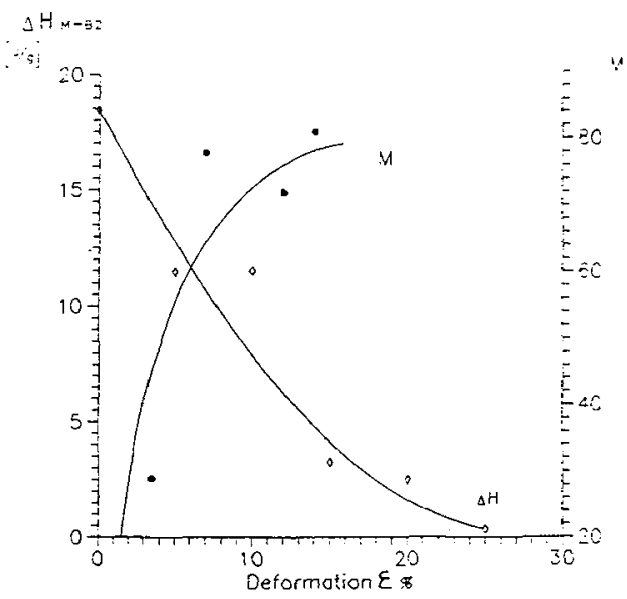

Fig. 2 Changes of enthalpy of reverse transformation and amount of martensite versus deformation degree

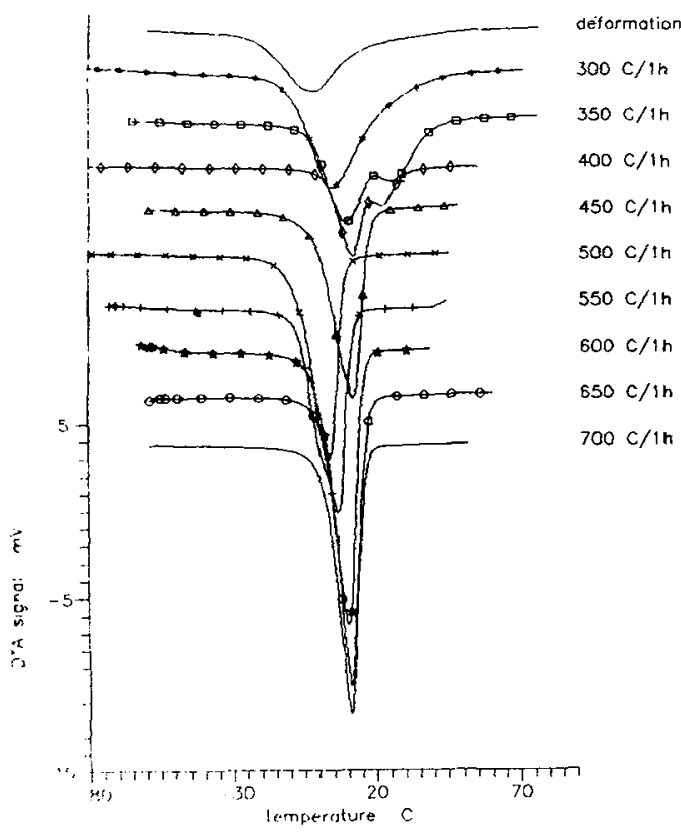

Fig. 4 The DTA curves obtained during heating of the alloy deformed by $10 \%$ 
This plot shows that the $\mathbf{M}_{\mathrm{s}}$ temperature decreases with the increase in deformation degree. Plastic deformation of the parent phase causes the creation of martensite in a quantity increasing with increase in deformation (Fig.2). After deformation by $20-25 \%$ the DTA cooling curve spreads to such a degree that the martensitic transformation is hardly visible. This indicates that the quantity of material undergoing transformation was small. More distinctive effects were observed on the heating curve. From the DSC curves obtained for these specimens the enthalpy of the reverse transformation $\Delta H_{M \rightarrow B 2}$ was calculated. Enthalpy changes versus deformation degree are shown in Fig.2.

\subsection{Effect of annealing}

The effect of annealing of the deformed alloy on the course of transformation was studied using the DTA method. Fig. 3 shows the DTA cooling curves for the alloy deformed by $10 \%$, and then annealed at various temperatures. The curve of the deformed alloy shows a strongly spread peak of martensite transformation on which overlapping of two effects may be distinguished, i.e. that of R-phase formation and, at lower temperatures, of martensite formation. Annealing of the alloy at $300^{\circ} \mathrm{C}$ causes distinct separation of those two effects. The separation becomes more visible when the annealing temperature increases up to $550^{\circ} \mathrm{C}$. Figure 3 also illustrates changes of the characteristic temperatures of both transformations versus the annealing temperature. Increase of annealing temperature up to $600^{\circ} \mathrm{C}$ causes disappearance of the R-phase transition peak.

The DTA curves obtained during cooling of specimens annealed at $400-550^{\circ} \mathrm{C}$ (except for

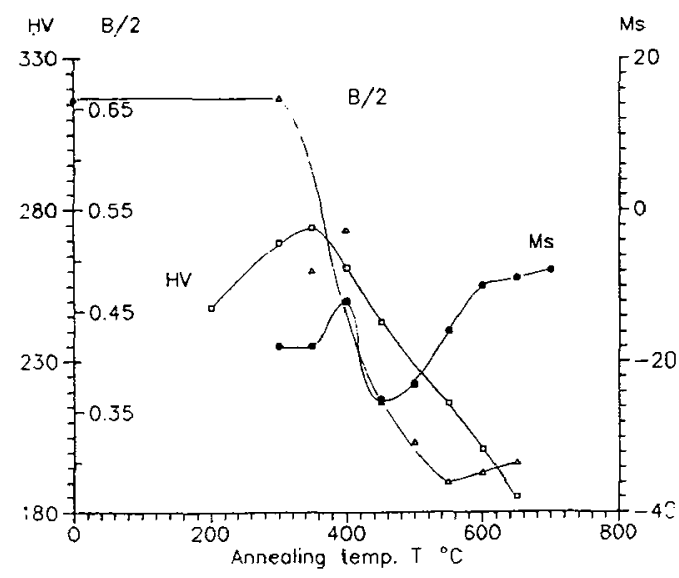

Fig. 5 Hardness, $M_{s}$ temperature and the half-width of the $110 \mathrm{~B} 2$ versus the annealing temperature for a specimen deformed by $10 \%$ the specimen annealed at $450^{\circ} \mathrm{C}$ ) show three distinct peaks, one from R-phase formation and two in the range of the martensitic transformation. Fig.4 presents the DTA curves obtained during heating of the same specimens. The reverse transformation for specimens annealed at $350^{\circ} \mathrm{C}$ and $400^{\circ} \mathrm{C}$ also consists of two distinct effects from the $M \rightarrow R$ and $R \rightarrow B 2$ transitions.

Fig. 5 shows the influence of annealing temperatures on half-width of the 110 B2 line for the alloy deformed by $10 \%$. The decrease in this parameter with increase in annealing temperature has a form similar to that for decrease in alloy hardness and is characteristic for the recovery process. Annealing at a temperature higher than $550^{\circ} \mathrm{C}$ does not cause any changes in this parameter. Disappearance of defects after annealing at these temperatures is also indicated by $M_{s}$ increase, whereas the $M_{s}$ decrease for specimens annealed at $400-500^{\circ} \mathrm{C}$ shows that besides lowering of defects density, there are other factors influencing the temperature. One of these could be the formation of dislocation cell structure. Thus, although total dislocation density decreases this structure inhibits the interface mobility and lower the $\mathbf{M}_{\mathrm{s}}$ temperature. Also the occurance of the R-phase transition in the specimen can influence the martensitic transformation in such a way that the $\mathrm{M}_{s}$ temperature decreases.

Transmission electron microscopy studies of alloy dislocation structure were carried out to investigated the influence of annealing on the course of transformations and on characteristic temperatures. Changes of the alloy structure versus temperature of annealing are shown on Fig. 6. A dislocation cell structure is visible. Dislocation density decreases with increase in annealing temperature. Annealing at $500^{\circ} \mathrm{C}$ causes partial recrystallization. Characteristic for this stage are areas where the polygonization process 
formed regular network of dislocations creating subgrains of low-angle boundaries. Annealing at $600^{\circ} \mathrm{C}$ causes full recrystallization of the deformed alloy. In order to explain the presense of the two peaks on the DTA curves in the martensitic transformation range, internal friction measurements were carried out. Results of these measurments obtained for alloy deformed by $10 \%$ and annealed at $400^{\circ} \mathrm{C}$ are shown on Fig. 7.

On the internal friction $\left(Q^{-1}\right)$ curve obtained during cooling there are three peaks. The first one, $P_{c 1}$ appears at the same temperature as the $\mathrm{B} 2 \rightarrow \mathrm{R}$ DTA peak. The fact that this peak is due to the $B 2 \rightarrow R$ transition is confirmed by the minimum on the curve of square of vibration frequency $\left(\mathbf{f}^{2}\right)$, which is proportional to the elastic modulus. The second internal friction peak $P_{c 2}$ does not have any equivalent either on
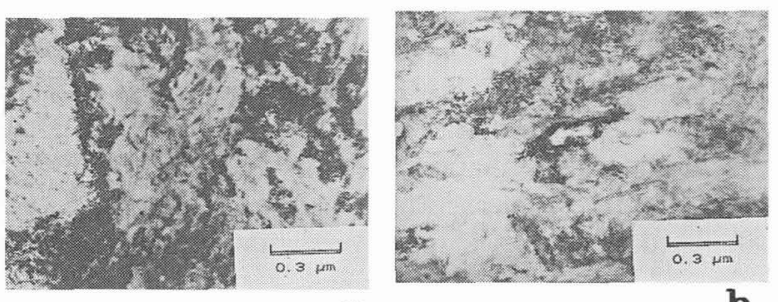

a
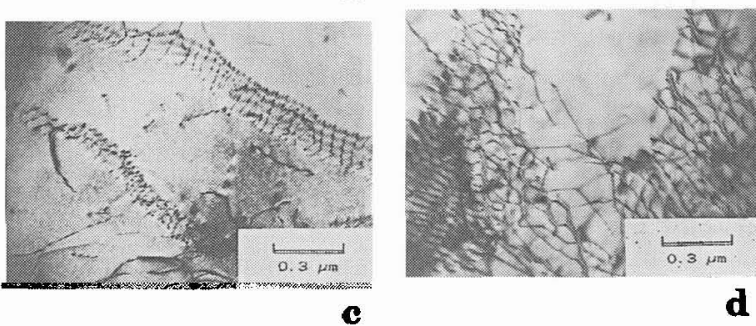

Fig. 6 Dislocation structure of the alloy deformed by $10 \%$ and annealed at $300^{\circ} \mathrm{C}(\mathrm{a}), 400^{\circ} \mathrm{C}(\mathrm{b}), 500^{\circ} \mathrm{C}(\mathrm{c}), 550^{\circ} \mathrm{C}$ (d) the DTA or on the $f^{2}$ curves. Thus it is not connected with the martensitic transformation. Its presence may be explained by relaxation of dislocations.

The third internal friction peak corresponds to the first martensite peak on the DTA curve. There is also a small but distinct minimum observed at this temperature on the $\mathbf{f}^{2}$ curve. The internal friction curve obtained during heating consists of two peaks which correspond to the temperatures at which peaks on the DTA curve arise as a result of $M \rightarrow R$ and $R \rightarrow B 2$ transformations. The second peak appearing on the DTA cooling curve close to that from the martensite transformation could be caused by interaction between the growing martensite plates and dislocation cell boundaries. For a plate to pass these boundaries additional energy is required and this may result in an additional peak on a DTA curve. This explains the fact that the peak is very clear for specimens annealed at $500^{\circ} \mathrm{C}$ and $550^{\circ} \mathrm{C}$ which contain a large number of subgrains formed by small-angle boundaries.

\section{Discussion}

Plastic deformation of the parent phase produces dislocations, the density of which can be estimated from the increase in half-width of an X-ray diffraction line for the phase. The higher the deformation degree, the wider is the $110 \mathrm{~B} 2$ line. The form of the curve representing the half-width versus deformation degree is similar to that for alloy hardness. The $\mathbf{M}_{s}$ temperature decreases with increase in dislocation density, which is due to the fact that the dislocations present obstacles for the moving interfaces. On the other hand, dislocations provide the places where the R-phase nucleates [9]. For small deformations the DTA curve obtained during cooling exhibits a peak corresponding to the R-phase formation. The higher the deformation, the more strain-induced martensite appears in the specimens, and the deformed B2 phase becomes stable. Thus the quantity of the parent phase undergoing thermal martensitic transformation decreases, as is proved by decrease in transformation heat obtained from the DSC measurements. Similar conclusions based on internal friction studies were presented in [10]. The annealing temperature has a vital influence on the course of transformations. Results presented on Figures 3 and 4 are in good agreement with data given in $[1,2]$. In the annealing range of $300-550^{\circ} \mathrm{C}$ the transformation occurs in two stages $\mathrm{B} 2 \rightarrow \mathrm{R} \rightarrow \mathrm{M}$ and after annealing at a temperature higher than $550^{\circ} \mathrm{C}$ only one stage transformation $\mathrm{B} 2 \rightarrow \mathrm{M}$ was observed. The same is true for the reverse transformation. Specimens annealed at low temperatures exhibited two stages of transformation course $M \rightarrow R \rightarrow B 2$ while with increase in annealing temperature overlapping of the two peaks is observed. The DTA curves presented on Fig. 3 exhibit two peaks in the range of the martensitic transformation for specimens annealed at 400,500 and $550^{\circ} \mathrm{C}$. Similar effects were observed for larger deformations. Todoroki and 
Tamura [1] obtained identical results on their DSC curves. Referring to the paper by Monasevich [11], they explained this effect as the formation of two types of martensite. Monasevich and Pascal observed continuous widening of the 111 martensite line during cooling. They concluded that monclinic martensite formed from the parent phase transforms to a triclinic and established deviations from orthogonality as $\alpha=0.3^{\circ}$ and $\gamma=0.5^{\circ}$. It is noteworthy that this effect was observed in alloys where no R-phase transformation occurred. Airoldli et al. [12], in specimens after cycling, observed two peaks from the martensite as well as the R-phase peak. They explained this effect in the same way as the authors of [1]. We obtained similar results for specimens after cycling [13], but we also observed that dislocations created during cycling form a cellular structure. Analysis of the DTA and internal friction curves (Fig.7) excluded the possibility of the formation of two types of martensite. Additionally, the results of X-ray studies, not presented in this paper, showed that the transformation sequence is $B 2 \rightarrow R \rightarrow B 19$ '. Thus we may conclude that the specific arrangements of dislocations are responsible for two peaks in the range of the martensitic transformation. Obstacles in the form of low-angle subgrain boundaries inhibit formation of the martensite in these places and cause the occurrence of a second peak at a lower temperature on the DTA and DSC curves.

Throughout the whole range of annealing temperatures, decrease in alloy hardness and in half-width of $110 \mathrm{~B} 2$ line is observed. This indicates that strengthening and lattice distortion of the alloy gradually disappears. Annealing at $500^{\circ} \mathrm{C}$ and higher does not change the density od defects any further, although alloy hardness still decreases. The $\mathbf{M}_{s}$ temperature for this annealing range increases slightly. After annealing in the temperature range $400-500^{\circ} \mathrm{C}$ the defects' density decreases, as does the $M_{s}$ temperature. Liu and McCormick [14] explained this fact basing on free energy changes analysis. If there occurs the $B 2 \rightarrow R$ transition, it shifts the next, $R \rightarrow M$ transformation to a lower temperature.

\section{REFERENCES}

[1] Todoroki T., Tamura H., Trans.Jap.Inst.Met. 28 (1987) 83.

[2] Abujudom D.N., Thoma P.E. Fariabi S., Mater. Sci. Forum 56-58 (1990) 565.

[3] Legresy J.M., Prandi B. Raynaud G.M., J.de Phys. IV, 1 (1991) C4-241.

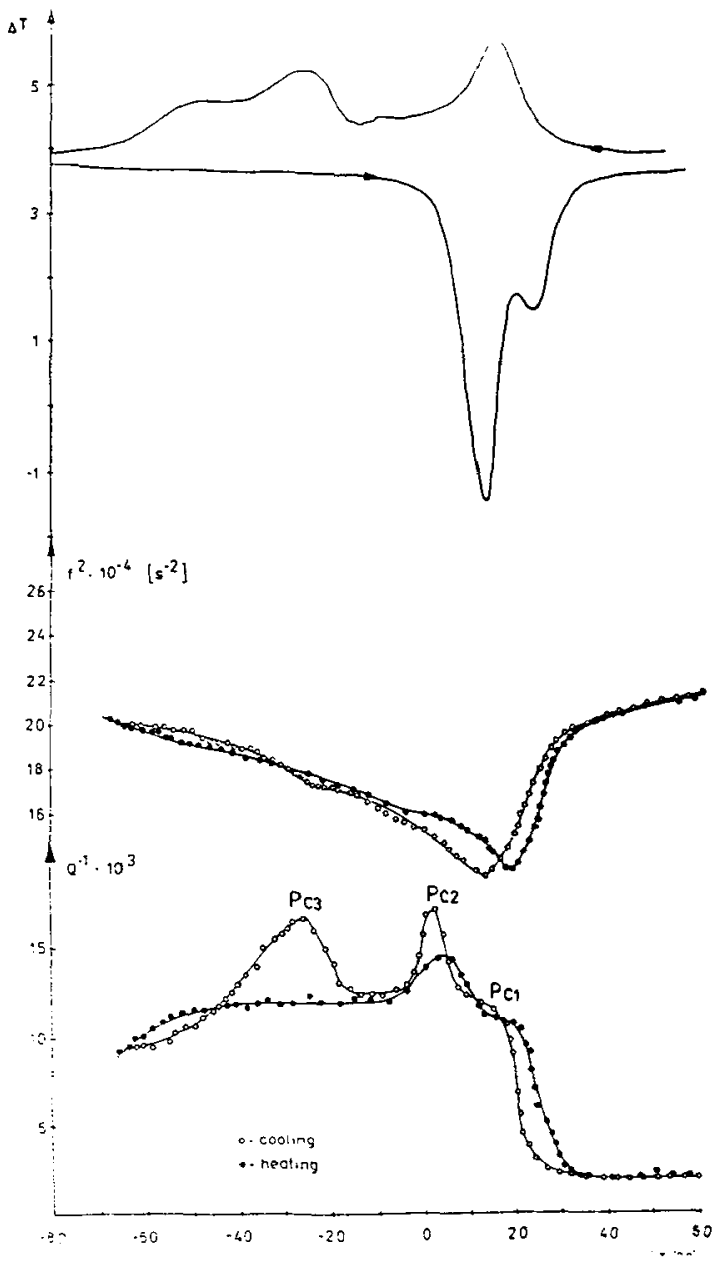

Fig. 7 The DTA curves (upper part) and the internal friction curves (lower part) for the alloy deformed by $10 \%$ and annealed at $400^{\circ} \mathrm{C}$ 
[4] Jinfang, Yuying C., Ming Z., Long S. Guansen Y., Mat.Res.Soc.Symp.Proc. 246, (1992) 283 Materials Research Society.

[5] Kao M.Y, Fariabi S., Thoma P.E., Ozkan H. and Cartz L., Mat.Res. Soc. Symp. Proc. 246, (1992) 225 Materials Research Society.

[6] Filip P., Rusek J. Mazanec K., Z.Metallk. 82 (1991) 488

[7] Thoma P.E., Kao M.Y., Fariabi S. and Abujudom D.N., Proc.Int.Conf.Mart.Trans., Monterey Inst. Adv. Studies (1993) 917 ed. by Wayman C.M. and Perkins J.

[8] Lin H.C. Wu S.K., Scripta Metall.Mater. 26 (1992) 59.

[9] Fukuda T., Saburi T., Doi K. and Nenno S., Mat.Trans. JIM, 33 (1992) 27.

[10] Lin H.C., Wu S.K., Chou T.S. Kao H.P., Acta Metall.Mater. 39 (1991) 2069.

[11] Monasevich L.A. Paskal Yu.J., Phys.Metals Metallogr. 49 (1980) 119.

[12] Airoldi G., Riva G. Rivolta B., Proc MRS - Int. Meeting on Adv. Mater., 9 (1989) 105.

[13] Stróż D., Bojarski Z., Ilczuk I., Lekston Z., Morawiec H., J.Mater.Sci. 26 (1991) 1741.

[14] Liu Y. McCormick P.G., Proc.Int.Conf.Mater.Trans. Monterey Inst.Adv. Studies (1993) 923, ed.by Wayman C.M. and Perkins J.

\section{ACKNOWLEDGMENTS}

This work was sponsored by the National Committee of Research through grant No 308639101 . 